\title{
Modelling of Fuzzy Based Handoff Decision Controller
}

\author{
Honey Soni', Vaishali Sood ${ }^{2}$, Anu Sheetal ${ }^{3}$ \\ Student, Electronics and Communication Department, GNDU, Gurdaspur, India ${ }^{1}$ \\ Assistant Professor, Electronics and Communication Department, GNDU, Gurdaspur, India ${ }^{2}$ \\ HOD, Electronics and Communication Department, GNDU, Gurdaspur, India ${ }^{3}$
}

\begin{abstract}
Handoff plays a pivotal role in wireless communication. It is responsible for providing seamless communication between Base Stations (BSs) when user moves from one cell to another. An accurate handoff decision is very hard because at the cell boundary Mobile Terminal (MT) experiences signal from serving BS as well as from neighbouring BSs. In this situation MT fluctuates between BSs due to which unnecessary handoffs occur and demean the signal quality, which is not acceptable. As an attempt in this direction, a fuzzy logic based controller is developed in this paper to reduce the number of unnecessary handoffs. With the advantage of feedback technology, this controller deals with the impact of indeterminable changes in the received signal strength (RSS) as the user moves. This controller helps to take an accurate decision only when handoff is needed. Simulation results reveal that the proposed controller reduces handoff probability and number of unnecessary handoffs.
\end{abstract}

Keywords: Fuzzy Logic, Handoff Decision, Cellular Networks, Received Signal Strength.

\section{INTRODUCTION}

The cellular network is the heart of the communication industry as it fulfils many requirements. Earlier mobile service uses only one powerful transmitter located at the highest spot in an area which communicate in a radius of up to 50 kilometres. But now the cellular concept organized the mobile telephone network in a different way. Many low-power transmitters are used throughout a coverage area rather than using only one powerful transmitter. Dividing a metropolitan region into one hundred different areas or cells with low-power transmitters increases the system capacity, which is essential to accommodate many users at a time. In this way the cellular concept utilizes variant low-power levels, which permit cells to be sized according to the subscriber density and requirement of a given area. The strength of cellular mobile communication is that as the population grows, cells can be added to accommodate the growth. The benefit of cellular network is to enable calls to be routed to and from the mobile phones. The problem in the cellular network arises when the mobile user traveled from one cell to another during call conversation, as chances of call dropping are more when a user crosses the boundary between adjacent cells. So handoff process is created to avoid dropping of a call.

Handoff is a process that occurs when the user moves out of the coverage area of a respective cell site. The handoff takes place when the BS to which MT is connected reassign control for user's call to a BS in neighbouring cell. When the BS is unable to maintain the call continuity, it informs all BSs in the surrounding cells about user's oncoming into their cells. Hence the call is taken by the new BS in which the user enters. This is how handoff is able to make interruption free communication between BSs during call conversation. Handoff is categorized as either soft handoff or a hard handoff. When there is a break in the connection before the establishment of a new connection with new BS is termed as hard handoff. In soft handoff, connection with the old BS releases after the connection with new BS is established. Hard handoff is used in Global System for Mobile (GSM) and soft handoff is implemented in Code Division Multiple Access (CDMA) systems. The term handoff used in this paper is referred to only hard handoff.

Seamless call continuity depends upon handoff decision. The handoff decision at the cell boundary is very challenging because when the user is between BSs, the MT switches the link with either BS due to which unnecessary handoffs occur and degrades the signal quality. The phenomenon of bouncing the link with MT back and forth by the BSs is called ping-ponging. To avoid the ping-pong effect, an accurate and efficient handoff decision should be taken by the network. Time of handoff decision is very critical as too early and late handoffs are not tolerable. The handoff decision is primarily determined by locally and relative short time factors such as RSS, bit error rate (BER), signal-to-interference noise ratio (SINR) etc. Undoubtedly, handoff decision algorithms based on RSS are most commonly used because RSS at the MT weakens deeply and rapidly as it moves. So in this paper, we have taken RSS as a parameter for the handoff decision and assume decentralized handoff procedure. Decentralizing the handoff decision, as in the case of the mobile assisted handoff, assist to attain fast forward handoff decision. With decentralizing, decisions are taken at BSs or Mobile Switching Center (MSC), but the MT cooperates in the search of objective BSs. This helps in conserving system resources. 
As the cell size reduces, numbers of handoffs increases since MT crosses more cells during a call. Thus, handoffs should occur quickly because delay in handoff may degenerate the quality of service below an acceptable level. To maintain the reliability in the system, handoffs must be performed successfully and infrequently and be unobserved to users. So to fulfill these requirements, threshold level is set as a minimum level for proper voice quality at the BS receiver. A fixed threshold value does not response to satisfactory result, because for the moving vehicles threshold level changes. So we need some advance techniques like neural networks, fuzzy logic system [1] and pattern recognition [2] that will yield variable threshold for the optimization of handoff. Moreover, variable threshold alleviates the ping-pong effect. Fuzzy logic system is preferred among other techniques as it gives precise decision for imprecise information and helps to resolve many ambiguities related to handoff decision.

This paper proposes a controller for the handoff decision based on fuzzy logic. Fuzzy Logic System (FLS) will use RSS as an input parameter and yield out threshold value for variant RSS. Operation of FLS is explained under the proposed work. The proposed controller results in providing accurate and fast handoff decision and reduces the number of unnecessary handoff.

\section{RELATED WORK}

Various techniques and algorithms have been proposed and developed to improve the handoff decision. It is sarcastic to design an efficient handoff algorithm offering seamless communication. The handoff procedure is categorized in three phases: initiation phase, decision phase and execution phase. Various handoff initiation methods based on signal level are relative signal level, relative signal level with threshold, relative signal level with hysteresis and relative signal level with threshold and hysteresis [3]. Malka et al. [4] evaluated handoff algorithms by a measure based on signal level. They have also proposed new call quality measures and introduced an off-line cluster-based computationally-simple heuristic algorithm that obtains a near optimal handoff sequence which can be used as a benchmark. The algorithm is further compared with an existing handoff algorithms and the tradeoff between number of handoffs and signal quality is identified. B. Senadji [5] proposed handoff decision algorithm for microcellular system. As handoff is optimal at cell boundary, therefore probability of handoff is maximum at these locations. So probability of handoff computed from RSSs is chosen as criterion for processing handoff from current BS to neighboring BS. The paper [6] gave a mathematical model for Wrong Decision Probability (WDP) and handoff probability (HP) which is used as criteria for the evaluation of handoff algorithm. For better performance WDP predication based handoff algorithm is also proposed as it reduces wrong decisions.

Smaoui et al. [7] introduced a scheme to reduce handoff delay using the concepts of RSS and threshold management for vertical handoff management protocol and also proposed a new secured IP assignment protocol to guarantee Quality-of-Service. Maksum et al. [8] discussed the evaluation of optimal value of handoff parameters by varying threshold and hysteresis values. For evaluation, they have used threshold with hysteresis method based on the tradeoff handoff parameters, namely (i) quality of signal level, (ii) number of handoffs and (iii) delay. They have concluded that large threshold value speed up the handoff and greater hysteresis values add to the delay. The complex handoff algorithms based on fuzzy logic system, neuro-fuzzy or neural network are validated approaches to solve handoff problems. Algorithms based on fuzzy logic have been used in decision making processes in many different areas, for example sensor networks [9], power management [10], health care [11], stock trading [12] and business forecasting [13]. In recent years, fuzzy logic based and related algorithms (for example, Adaptive Neural Fuzzy Inference System (ANFIS)) have attracted the researchers for the design of intelligent handoff decision systems. Fuzzy logic based handoff algorithm is presented in [14] for the reliable communication in microcellular system. Handoff is based on the ratio of slopes of normal signal loss to the actual signal loss, since decrease in RSS is well monitored by determining ratio of slopes of expected normal signal and the actual signal. Kwong et al. [15] proposed newer approach using ANFIS where the training element is integrated into the existing fuzzy handoff algorithm. The performance metrics used as an input to fuzzy Inference System (FIS) are RSS and BER.

Fatma et al. [16] optimizes the vertical handoff procedure by combining RSS and service type. At the same time, fuzzy logic system was used to manifest the cell selection. But the RSS threshold for vertical handoff decision is fixed due to which ping-pong effect cannot be alleviated. A handoff algorithm termed as Fuzzy Controller for Handoff Optimization is designed in [17] using fuzzy concepts. The proposed algorithm is better than traditional algorithms as it exploits entrancing features of various existing algorithms and imparts more capabilities like providing adaptation to the dynamic environment. The proposed algorithm eliminates the corner effect problem by dynamically changing the threshold and hysteresis values with the change in the RSS and the velocity of the MT. Barolli et al. [18] proposed fuzzy logic based a new handoff system that avoid ping-pong effect and offers an accurate handoff decision. Fuzzy logic is used to operate with imprecision data and can model non-linear functions. Three parameters have been used in the proposed system for handoff decision: the change of signal strength of the present BS, signal strength from the neighbor BS and the distance between MT and BS. A fuzzy logic control based QoS management scheme for Wireless Sensor/Actuator Networks (WSAN) is developed in [19] to support QoS in dynamic and unpredictable environments. This scheme deals with the affect of sporadic changes in traffic load of WSANs with the help of feedback technology. Fuzzy logic controller is used to adjust sampling period dynamically to the deadline miss ratio linked with data transmission from 
the sensor to the actuator, so that deadline miss ratio is maintained at desired level to achieve QoS.

\section{PROPOSED WORK}

In this paper, fuzzy logic based decision making handoff controller is designed to achieve optimal handoff decision for cellular networks, shown in fig. 1. In proposed controller, MT measures RSS from the current BS. Here, fuzzy logic system is employed to adjust the threshold value dynamically using RSS so that threshold level is maintained at desired level to avoid call dropping. The MT reports the threshold measures to the BS. In the proposed model, transfer function is used to specify the BS. Lower threshold level indicates weak signal strength at MT, so the chances of call-dropping are more. Hence, lower value of threshold designate to pass the control to another BS. For optimal handoff decision, RSS threshold value should be accurate. For this reason, feedback control is used for the better and correct handoff decision. With respect to previous threshold value from the BS and RSS, the controller generates new threshold value using fuzzy logic control algorithm. On the basis of this new threshold value handoff decision is taken by the BS.

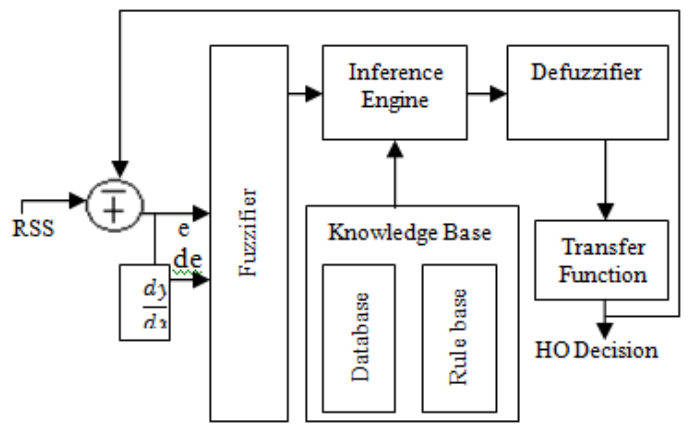

Fig. 1: Architecture of Fuzzy based Handoff Decision Controller

We have used second order transfer function for the BS. The supremacy of transfer functions is that they offer a convenient form for analyzing and manipulating complex feedback systems, since it provides a mapping between inputs and their corresponding outputs. It also makes it possible to express the changes in a system because of modeling error, which is essential when discussing sensitivity to variations, as in handoff case. The results shown in next section have described how the proposed controller minimizes the number of handoffs and handoff probability.

\section{A. Fuzzy Logic System Design}

Fuzzy logic system offers an effective and flexible way to reach at a definite conclusion based on imprecise or incomplete input information. Therefore, it can easily deal various uncertainties rises in cellular networks, such as sudden fall in signal strength, ping-pong effect and unpredictable change in the position of MT. The function of fuzzy logic system in the proposed work is to determine the threshold value based on previous threshold and RSS. Fuzzy logic system consists of four main components: fuzzifier, knowledge base (database and rule base), inference engine and defuzzifier. As can be seen in figure 1 , there are two inputs, i.e., error $\mathrm{e}(\mathrm{k})=\mathrm{RSS}-$ previous threshold value and change in error $\mathrm{de}(\mathrm{k})=\mathrm{e}(\mathrm{k})-\mathrm{e}(\mathrm{k}-1)$ and one output that is threshold th(k). The fuzzifier converts numeric inputs $\mathrm{e}(\mathrm{k})$ and $\mathrm{de}(\mathrm{k})$ into fuzzy sets characterizing linguistic variables $\mathrm{E}$ and $\mathrm{DE}$. The inference engine then applies a predetermined set of IFTHEN fuzzy rules in the rule base related to linguistic variables, and the fuzzy sets of the output linguistic variable $\mathrm{TH}$ are produced. These fuzzy sets are then aggregated using inference method. Lastly, the defuzzifier converts the aggregated fuzzy value back to the numeric value th(k).

TABLE 1: RANGES OF INPUT ERROR, CHANGE IN
ERROR AND THRESHOLD
\begin{tabular}{|c|c|c|}
\hline Parameters & Ranges $(\mathbf{d B m})$ \\
\hline & from & to \\
\hline error (e) & -3 & 3 \\
\hline change in error (de) & -1 & 1 \\
\hline threshold (th) & -92 & -86 \\
\hline
\end{tabular}

For the operation of fuzzy logic system, deciding ranges for input and output parameters are necessary. Because the size of cell is small, RSS limits the cell boundary at -100 $\mathrm{dBm}$. The ranges for input and output parameters are described in Table 1. The threshold error will vary from $3 \mathrm{dBm}$ to $3 \mathrm{dBm}$. Change in error must be smaller than error, so it is taken from $-1 \mathrm{dBm}$ to $1 \mathrm{dBm}$. At the cell boundary, $-100 \mathrm{dBm}$ to $-94 \mathrm{dBm}$ is the level for requesting a handoff because at this level signal quality degrades and requires a handoff immediately. Therefore, range for threshold is decided from $-92 \mathrm{dBm}$ to $-86 \mathrm{dBm}$ as handoff ambiguity arises between these ranges.

Now, we will discuss membership functions for both input and output linguistic variables. The linguistic values for the input linguistic variables $\mathrm{E}$ and $\mathrm{DE}$ are $\{\mathrm{NL}, \mathrm{LS}, \mathrm{MR}$, $\mathrm{PM}\}$ and $\{\mathrm{NG}, \mathrm{ZE}, \mathrm{PO}\}$, respectively. NL, LS, MR, PM, $\mathrm{NG}, \mathrm{ZE}$ and $\mathrm{PO}$ represent negative less, less, more, positive more, negative, zero and positive, respectively. Similarly, the set of linguistic

TABLE 2: FUZZY RULES

\begin{tabular}{|c|c|c|c|}
\hline DE & NG & ZE & PO \\
\hline NL & -- & HI & -- \\
\hline LS & HR & ME & LR \\
\hline MR & ME & LO & -- \\
\hline PM & -- & LR & -- \\
\hline
\end{tabular}

values for TH is $\{\mathrm{LR}, \mathrm{LO}, \mathrm{ME}, \mathrm{HI}, \mathrm{HR}\}$ where LR, LO, ME, HI and HR represent lower, low, medium, high and higher, respectively. The maximum number of fuzzy rules formed from defined input linguistic values should be 12 
but in this paper, only 7 rules are formed, presented in Table 2. These rules are formed, taking into account the precise knowledge of handoff occurrence in cellular networks. Lower value of $\mathrm{E}$ and DE maintains threshold value higher while larger values of e and de force the network for the handoff. The '- ' mark indicates that rules corresponding to the combination of $\mathrm{E}$ and $\mathrm{DE}$ have not been used in the proposed controller. Fuzzy rules encode knowledge about a system of the form:- IF (a set of conditions) are satisfied THEN (a set of consequents) can be inferred [20]. Fuzzy IF-THEN rules in the proposed system are coded in the form:-

IF $\left(x_{1}\right.$ is $\tilde{A}_{1}, x_{2}$ is $\tilde{A}_{2}, \ldots \ldots, x_{n}$ is $\left.\tilde{A}_{n}\right)$ and $\left(y_{1}\right.$ is $\tilde{B}_{1}, y_{2}$ is $\tilde{\mathrm{B}}_{2}, \ldots \ldots, \mathrm{y}_{\mathrm{n}}$ is $\left.\tilde{\mathrm{B}}_{\mathrm{n}}\right) \operatorname{THEN}\left(\mathrm{z}_{1}\right.$ is $\tilde{\mathrm{C}}_{1}, \mathrm{z}_{2}$ is $\tilde{\mathrm{C}}_{2}, \ldots \ldots, \mathrm{z}_{\mathrm{n}}$ is $\tilde{\mathrm{C}}_{\mathrm{n}}$ )

where linguistic variables $x_{i}, y_{j}, z_{k}$ take values of fuzzy sets $A_{i}, B_{j}$ and $C_{k}$, respectively and $n$ represents the number of rules. The Max-Min method is followed for the inference mechanism. In the Max-Min inference method, the fuzzy operator AND (intersection) implies that the minimum value of the conditions is taken:-

$\mu_{\mathrm{A}} \operatorname{AND} \mu_{\mathrm{B}}=\min \left\{\mu_{\mathrm{A}}, \mu_{\mathrm{B}}\right\}$

for any two membership values $\mu_{A}$ AND $\mu_{B}$ of the fuzzy

sets A, B, respectively. All the formulated rules are aggregated using the union operator, thus generating the output fuzzy set C.

$$
\mathrm{C}=\mathrm{C}_{1} \cup \mathrm{C}_{2} \cup \mathrm{C}_{3} \ldots \ldots \cup \mathrm{C}_{\mathrm{n}}
$$
where, $\mu_{\mathrm{C}}(\mathrm{z})=\max \left(\mu_{\mathrm{C} 1}(\mathrm{z}), \mu_{\mathrm{C} 2}(\mathrm{z}), \ldots \ldots, \mu_{\mathrm{Cn}}(\mathrm{z})\right), \forall \mathrm{z} \epsilon$ $\mathrm{Z}$

where $\mathrm{Z}$ is the universe of discourse. The membership functions used in fuzzy logic system are triangular and trapezoidal as they are simple and easy to implement. However, less number of rules is organized to reduce computational cost. In the defuzzifier, the most popular centre of gravity method is used to produce a real number in the range of the output. The strength of centre of gravity method is that it gives an accurate result based on the weighted values of numerous output membership functions and is given by:-

$$
\mathrm{z}^{*}=\frac{\int \mu(\mathrm{z}) \mathrm{zdz}}{\int \mu(\mathrm{z}) \mathrm{dz}}
$$

where $z^{*}$ is the defuzzified output, $\mu(z)$ is the aggregated membership function and $\mathrm{z}$ is the output variable.

\section{RESULTS AND DISCUSSION}

To evaluate the performance of proposed controller, simulations are conducted in this section. Fuzzy logic toolbox and MATLAB simulator is used to obtain the results. The MT measures the RSS from BS and applied it to the fuzzy logic system to yield threshold value by using previous threshold value. The resultant threshold value will state the requirement of handoff decision. The MT needs handoff when the threshold level turns down. At that instant, MT will inform BS and request for handoff. The results of proposed controller are shown in figures below.

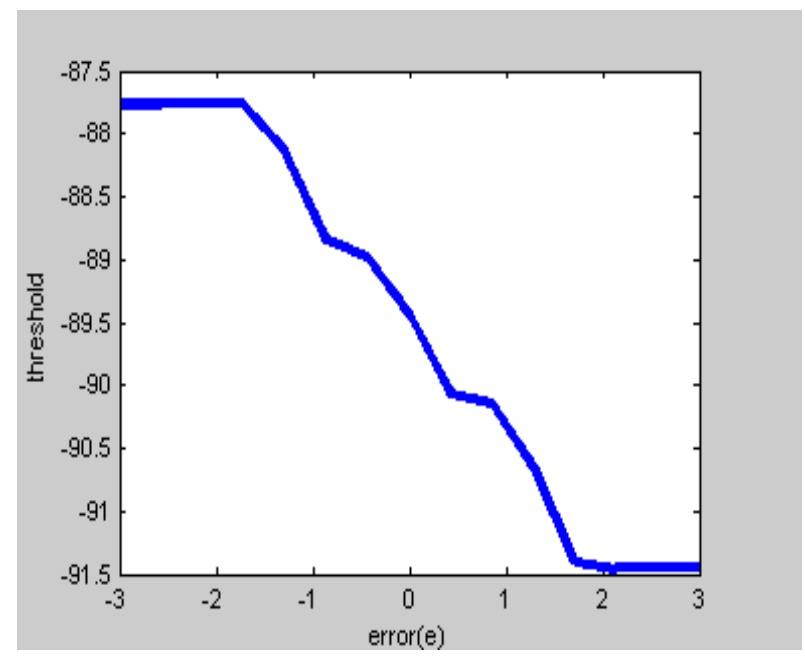

Fig. 2: Variation of threshold with respect to error

Fig. 2 is the output of fuzzy logic system that depicts the variation of threshold with respect to error. Error is the difference between RSS and previous threshold that is feedback to the fuzzy logic system. Less the error, lesser will be threshold, therefore less chances of handoff. For example, if RSS is $-87 \mathrm{dBm}$ and previous threshold is -91 $\mathrm{dBm}$, then an error $=-87 \mathrm{dBm}-(-91 \mathrm{dBm})$ will be $3 \mathrm{dBm}$, which is very high. High value of error is not acceptable, so threshold value decreases to assist handoff decision, which is also can be seen in fig. 2 .

Fig. 3 shows the variation of threshold with respect to change in error. Change in error is the difference between the error at current and previous instant. For example, if error for last instant is $3 \mathrm{dBm}$ and $2 \mathrm{dBm}$ for current instant, then de $=(3-2)$. $\mathrm{dBm}$ will be $1 \mathrm{dBm}$, which is not tolerable. This depicts that the MT changes its position rapidly due to which signal gets weak. This necessitates a handoff control and therefore threshold value should be low for this case as can be seen in fig. 3. The fig. demonstrates that with the increase in de, the threshold value decreases or vice-versa.

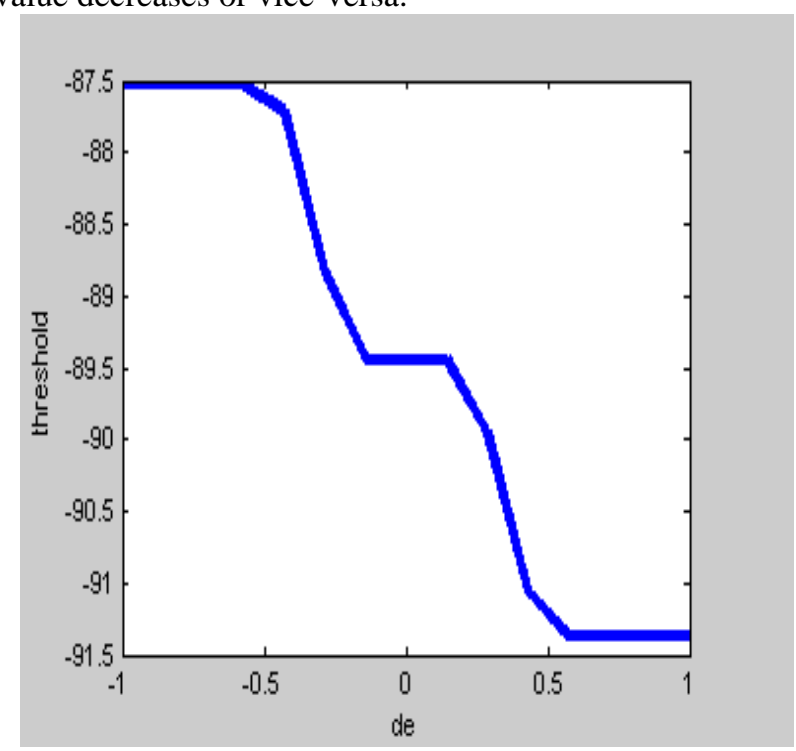

Fig. 3: Variation of threshold with respect to change in error 
Fig. 4 describes the relation of error and change in error with threshold. The relation can be explained as: when e and de is less then threshold value increases and when both are more then threshold decreases to facilitate the handoff. This will be more apprehensible with an example, when e is $-2 \mathrm{dBm}$ and de

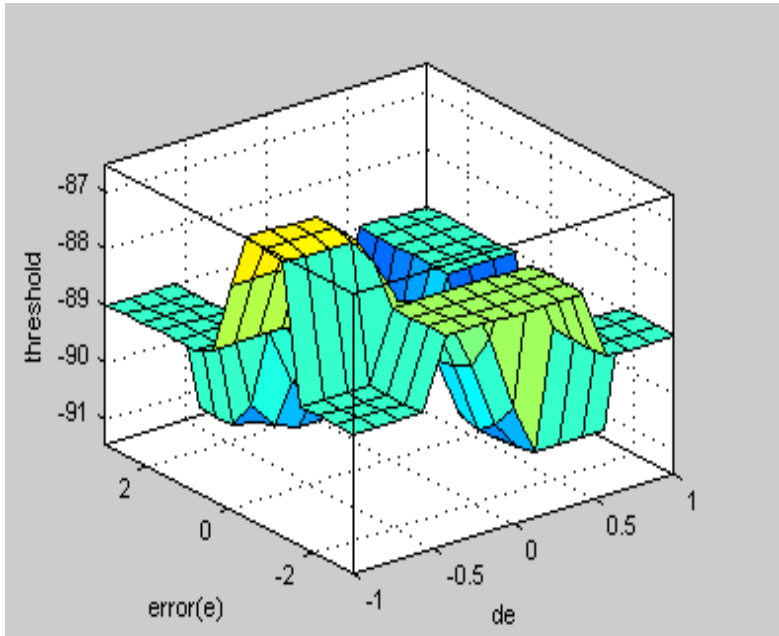

Fig. $4: 3$-D surface view relating error and change in error with threshold

is $0 \mathrm{dBm}$, then the threshold corresponding to these values is $-88 \mathrm{dBm}$. This combination of e and de is permissible since error is less and change in error lies in between negative and positive values. Therefore threshold should be high to avoid handoff which is depicted in the fig. above. The performance of the controller in this paper is determined by examining the handoff probability and number of handoffs. Fig. 5 illustrates an inverse relationship of RSS and number of handoffs. Result shows that number of handoff increases as the RSS decreases. It is clear in fig. 5 that upto $-100 \mathrm{dBm}$ (maximum range for microcellular network), number of handoffs is less while after that handoff number increases.

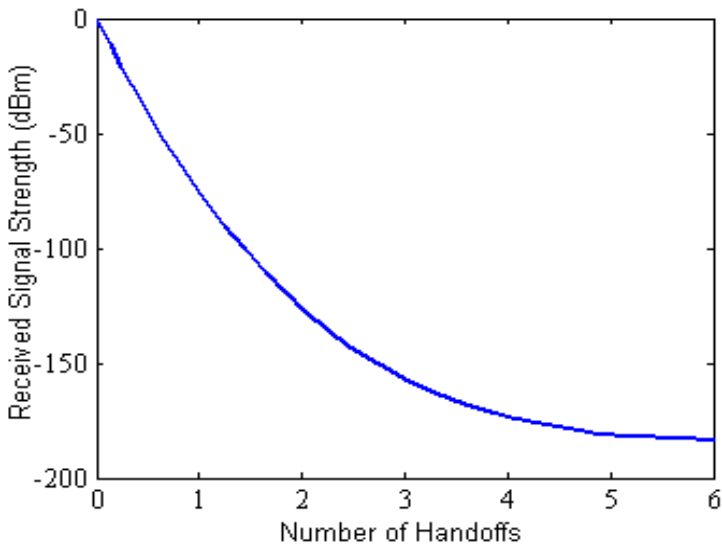

Fig. 5: Graph shows number of handoffs with varying RSS

Thus, the proposed model performs better and able to reduce number of handoffs. On the other hand, handoff probability states about the occurrence of handoff with respect to RSS values. It is evident from fig. 6 that as the RSS decreases, handoff probability increases. It is mentioned in the above section that at the range between $100 \mathrm{dBm}$ to $-94 \mathrm{dBm}$ of RSS, the signal gets weak in the microcellular network and requires handoff which can be viewed in fig. 6 that below $-92 \mathrm{dBm}$ the handoff probability is very high. Thus, the proposed model gives an accurate handoff decision, avoids unnecessary handoffs and address ping-pong effect.

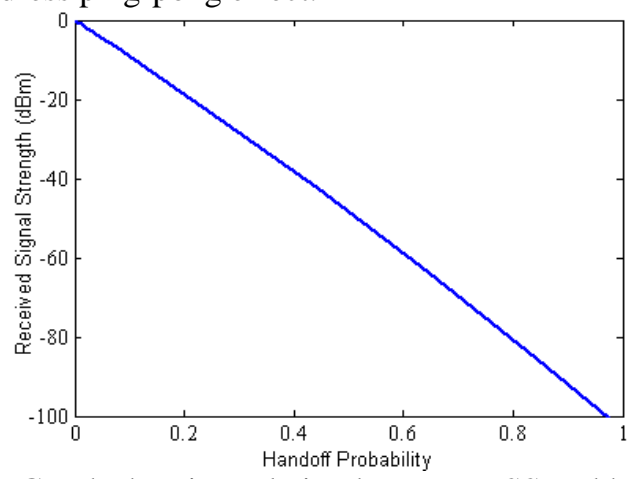

Fig. 6: Graph showing relation between RSS and handoff probability

Thus, the proposed model performs better and able to reduce number of handoffs. On the other hand, handoff probability states about the occurrence of handoff with respect to RSS values. It is evident from fig. 6 that as the RSS decreases, handoff probability increases. It is mentioned in the above section that at the range between $100 \mathrm{dBm}$ to $-94 \mathrm{dBm}$ of RSS, the signal gets weak in the microcellular network and requires handoff which can be viewed in fig. 6 that below $-92 \mathrm{dBm}$ the handoff probability is very high. Thus, the proposed model gives an accurate handoff decision, avoids unnecessary handoffs and address ping-pong effect.

\section{CONCLUSION}

In this paper, modelling of fuzzy logic based handoff decision controller is presented. Fuzzy logic is used because it is suitable for an analysis for environment of uncertainty. This controller aims at reducing the unnecessary handoffs and provides seamless communication by taking handoff decision timely. For the handoff decision, controller uses feedback technology and computes threshold value dynamically using RSS and previous threshold. Different concept used in the proposed work is the transfer function for the communication between MT and BS. Simulation results show that the proposed controller is effective as it is able to take handoff decision only when it is necessary and alleviates the pingpong effect. The benefit of this controller is its simplicity and low computational cost as less number of rules is used. For the future work, we will predict RSS and priority based handoff decision algorithms will be employed for fast and optimal handoff.

\section{ACKNOWLEDGMENT}

We would like to express gratitude to GNDU faculty for all the help they have provided.

\section{REFERENCES}

[1]. M. S. Dang, A. Prakash, D. K. Anvekar, D. Kapoor, and R. Shorey, "Fuzzy logic based handoff in wireless networks," in IEEE Vehicular Technology Conference, vol. 3, May 2000, pp. 2375 2379 . 


\section{International Journal of Advanced Research in Computer and Communication Engineering} Vol. 4, Issue 5, May 2015

[2]. A. Majlesi and B. H. Khalaj, "An adaptive fuzzy logic based handoff algorithm for interworking between WLANs and mobile networks," in IEEE International Symposium of Personal, Indoor and Mobile Radio Communications, vol. 5, Sept. 2002, pp. $2446-$ 2451.

[3]. Marichamy, P., Chakrabarti, S., "On Threshold Setting and Hysteresis Issues of Handoff Algorithms", in Proc. IEEE Personal Wireless Communication Conf., 1999, pp. 436-440.

[4]. Malka N. Halgamuge, Hai Le Vu, Kotagiri Ramamohanarao and Moshe Zukerman, "Signal-Based Evaluation of Handoff Algorithms", IEEE Communications Letters, vol. 9, no. 9, 2005, pp. 790-792.

[5]. B. Senadji, "A New Handover Decision Algorithm For Microcellular Systems", in Proc. IEEE, 1997, pp. 314-318.

[6]. C. Chi, X. Cai, R. Hao and F. Liu, "Modeling and Analysis of Handover Algorithms", in Proc. IEEE GLOBECOM, 2007, pp. 4473-4477.

[7]. I. Smaoui, F. Zarai, and L. Kamoun, "Vertical handoff management for next generation heterogeneous networks", ITI 5th International Conference on Information and Communications Technology (ICICT), 2007, pp.19-25.

[8]. Maksum Pinem, Eddy Marliant, Nasruddin M. N. and Fitri Arnia, "Evaluation of the Optimal Handoff Parameters using the Method of Threshold with Hysteresis", International Journal of Engineering Research \& Technology (IJERT), Vol. 3, issue 1, 2014, pp. 22192223 .

[9]. Lukic, M., Ja.nicijevic, N., Mezei, I., "Improved decision making in WSN based on localized auctions and fuzzy logic," in Proc. of 19th Telecommunications Forum (TELFOR), 2011, pp.238-241.

[10]. Sanaye-Pasand, and M., Jafarian, P., "An adaptive decision logic to enhance distance protection of transmission lines," IEEE Transactions on Power Delivery, vol.26, no.4, 2011, pp.2134-2144.

[11]. Seker, H.; Odetayo, M.O.; Petrovic, D.; Naguib, R.N.G.; , “A fuzzy logic based-method for prognostic decision making in breast and prostate cancers," IEEE Transactions on Information Technology in Biomedicine, vol.7, no.2, 2003, pp.114-122.

[12]. Esfahanipour, A., Mardani, P., "An ANFIS model for stock price prediction: The case of Tehran stock exchange," in Proc. of Int. Symposium on Innovations in Intelligent Systems and Applications (INISTA), 2011, pp.44-49.

[13]. Ben Ghalia, M., Wang, P. P., "Intelligent system to support judgmental business forecasting: the case of estimating hotel room demand," IEEE Transactions on Fuzzy Systems, vol.8, no.4, 2000, pp.380-397.

[14]. G. M. Mir, N. A. Shah and Moinuddin, "Decentralized Handoff for Microcellular Mobile Communication System using Fuzzy Logic", World Academy of Science, Engineering and Technology, vol. 3, no. 2, 2009, pp. 663-667.

[15]. Chiew Foong Kwong, Teong Chee Chuah and Sze Wei Lee, "Adaptive Network Fuzzy Inference System (ANFIS) Handoff Algorithm", International Journal of Network and Mobile Technologies, vol.1, issue 2, 2010, pp. 54-59.

[16]. Fatma T and Muhammed S, "On the vertical handoff decision for wireless overlay networks", Proc. of the 7th IEEE International Symposium on Computer Networks (ISCN'06), 2006, pp. 111-115.

[17]. Pooja Dhand and Parwinder Dhillon, "Handoff Optimization for Wireless and Mobile Networks using Fuzzy Logic", International Journal of Computer Applications, vol. 63, no. 14, 2013, pp. 31-35.

[18]. Leonard Barolli, Fatos Xhafa, Arjan Durresi and Akio Koyama, "A Fuzzy-based Handover System for Avoiding Ping-Pong Effect in Wireless Cellular Networks", in Proc. IEEE International Conference on Parallel Processing - Workshops (ICPP-W), 2008, pp. 135-142.

[19]. Feng Xia, Wenhong Zhao, Youxian Sun and Yu-Chu Tian, "Fuzzy Logic Control Based QoS Management in Wireless Sensor/Actuator Networks", Published in Sensors, vol. 7, no.12, 2007, pp. 3179-191.

[20]. S. Rajasekaran and G.A. Vijayalakshmi Pai, "Neural Networks, Fuzzy Logic, and Genetic Algorithms (Synthesis and Applications)", Eastern economy edition, PHI, ISBN-81-203-21863 . 